\title{
Extended High Frequency Audiometry for Revealing Sudden Sensory Neural Hearing Loss in Acute Tinnitus Patients
}

\author{
Rani Abu-Eta1ำ Haim Gavriel ${ }^{1}$ Jacob Pitaro $^{1}$ \\ ${ }^{1}$ Department of Otolaryngology-Head and Neck Surgery, Shamir \\ (Previously Assaf Harofeh) Medical Center, Zerifin, Israel \\ Int Arch Otorhinolaryngol 2021;25(3):e413-e415.
}

\begin{abstract}
Address for correspondence Rani Abu-Eta, MD, Department of Otolaryngology-Head and Neck Surgery, Shamir (previously Assaf Harofeh) Medical Center, Zerifin 70300, Israel

(e-mail: rabuita@gmail.com).
\end{abstract}

\begin{abstract}
Introduction The measurement of extended high-frequency (EHF) audiometry has become more popular recently, mainly in connection with ototoxicity and noiseinduced hearing loss. New-onset tinnitus evaluation includes a standard hearing test that shows no pathology.

Objective The aim of the present study was to evaluate the possibility that acute tinnitus is essentially connected to sudden sensory neural hearing loss (SSNHL), by utilizing EHF audiometry in cases in which standard audiometry for frequencies between $250 \mathrm{~Hz}$ to $8 \mathrm{kHz}$ is within normal limits.

Keywords

- extended high frequency audiometry

- sudden sensory neural hearing loss

- tinnitus

Methods A retrospective study was conducted between January 2009 and May 2014 that included all patients presenting with acute tinnitus and normal standard audiometry. All patients underwent EHF audiometry and were treated accordingly.

Results Thirty-two patients with acute tinnitus and asymmetric sensorineural hearing loss on EHF audiometry were identified. The average deltas between the ears were between 9.2 and 33dB (worse in the affected ear).

Conclusion Extended high-frequency audiometry up to $20,000 \mathrm{~Hz}$ should be performed in all patients with acute tinnitus and standard audiometry within normal limits.
\end{abstract}

\section{Introduction}

The most common method for assessment of hearing loss is pure-tone audiometry, which is performed at frequencies of 250 to $8,000 \mathrm{~Hz}$. Recently, there has been an increasing interest in the measurement of auditory thresholds above $8000 \mathrm{~Hz}$ (the extended high frequency [EHF]). The measurement of the EHF range is facilitated by the development of audiometers capable of delivering sounds with sufficient pressure levels and earphones that can optimally transmit sound through the ear canal. ${ }^{1}$ The EHF audiometry, although not validated for clinical use, is used to monitor patients under a high risk of hearing loss. Although mainly used in research, EHF audiometry is also used in some clinical protocols to detect early changes to the auditory thresholds and to plan early intervention when possible. The clinical utility of EHF audiometry has newly been proposed for the monitoring of ototoxicity-induced hearing loss and the adverse effects of noise. The use of EHF audiometry to detect ototoxicity prior to involvement of the frequencies important for verbal communication has been reported. ${ }^{2}$ The results of this study strengthen the need to serially monitor auditory thresholds, especially in the high-frequency range, of patients receiving ototoxic drugs. The use of EHF received

February 4, 2020

accepted

May 6, 2020

published online

September 30, 2020
DOI https://doi.org/

10.1055/s-0040-1713921. ISSN 1809-9777.

\footnotetext{
(c) 2020. Fundação Otorrinolaringologia. All rights reserved.

This is an open access article published by Thieme under the terms of the Creative Commons Attribution-NonDerivative-NonCommercial-License, permitting copying and reproduction so long as the original work is given appropriate credit. Contents may not be used for commercial purposes, or adapted, remixed, transformed or built upon. (https://creativecommons.org/ licenses/by-nc-nd/4.0/)

Thieme Revinter Publicações Ltda., Rua do Matoso 170, Rio de Janeiro, RJ, CEP 20270-135, Brazil
} 
audiometry to detect noise-induced hearing loss is less established. Extended high frequency audiometry has been suggested as being more sensitive to detect noise-induced hearing loss in noisy occupational environments, especially in younger workers. ${ }^{3}$ However, other studies have concluded that EHF audiometry has no additional value in predicting noise-induced hearing loss. ${ }^{4-6}$ It is not unusual for patients with acute tinnitus to have standard audiometric evaluation that is within normal limits. However, these patients may have EHF hearing loss that may be missed on standard 250 to $8,000 \mathrm{~Hz}$ audiometry. In this study, we sought to examine cases of acute tinnitus with normal hearing between 250 to $8,000 \mathrm{~Hz}$, and assess whether they may represent SSNHL based on EHF audiometry results.

\section{Materials and Methods}

The present study was approved by the institutional review board.

In a retrospective study conducted between January 2009 and May 2014 at the Otolaryngology Head and Neck Surgery Department of the Assaf Harofeh Medical Center, all patients presenting with acute tinnitus were identified. Patients of all ages were included in our study. We included all patients who presented with: (1) previously normal hearing as reported by the patient; (2) audiometry confirmation of a $30-\mathrm{dB}$ hearing loss at 3 consecutive frequencies within less than 72 hours from symptom evolvement; (3) absence of previous or active ear infections; (4) no previous history of noise exposure or phonal trauma; (5) no previous history of ototoxic medications; (6) no family history of hearing loss; and (7) no history of chronic tinnitus. All patients were examined in the Emergency Department and were subjected to careful ear examination to identify any abnormalities that might interfere with hearing, such as a tympanic membrane perforation or other middle ear abnormalities. All participants completed an audiologic evaluation including pure tone audiometry for the range $250 \mathrm{~Hz}$ to $8,000 \mathrm{~Hz}$. In patients with normal symmetric hearing, the examination was extended to $20,000 \mathrm{~Hz}$. Conventional frequency audiometry and EHF audiometry were conducted in a sound-treated room (MOD. AP30). The instrument used for the study was the Amplaid A319 high frequency clinical audiometer (Amplifon. Milan, Italy) with standard TDH-49 headphones (Telephonics, New York, United states) used for the frequency range of 250 to $8,000 \mathrm{~Hz}$, and Sennheiser HDA200 circumaural phones (Braunscweig, Germany) used to test the extended high frequency 8,000 to $20,000 \mathrm{~Hz}$ range. Measurements were made with an ascending-descending technique, in 5-dB steps in both conventional and EHF audiometry. All thresholds were calculated in dB HL. Audiologic tests were analyzed for asymmetric sensory neural hearing loss. Although audiometry confirmation of a 30-dB hearing loss at 3 consecutive frequencies within less than 72 hours from symptom evolvement was the definition of SSNHL in our study, a $10 \mathrm{~dB}$ difference between the 2 ears was recorded as asymmetric hearing. Audiograms were performed before commencing therapy. All patients with asymmetric sensorineural hearing loss were treated with an oral steroid (oral prednisone $1 \mathrm{mg} / \mathrm{kg}$ for 7 days). In addition, average bone conduction thresholds in EHF were compared with reference thresholds. ${ }^{1}$

\section{Results}

Our study population included 28 patients who complained of having new-onset tinnitus. The study consisted of 16 men and 12 women with a mean age of 41.5 years (10-69 years). Three children were identified during the study period. Comorbidities were detected in 7 patients, including: hypertension in 4 (14.5\%) patients, hyperlipidemia in 2 (7\%), and cerebrovascular accident in 1 (3.5\%). The duration range of tinnitus prior to admission was 0 to 7 days (mean: 3.3 day). Nineteen (67\%) patients complained of having ipsilateral hearing loss sensation, 10 (35\%) patients had dizziness, and 14 (50\%) had aural fullness. All patients met the inclusion criteria and did not complain of other symptoms. The right ear was involved in 16 (57\%) patients and the left in 12 (43\%). All patients had a normal otoscopic examination. All patients had normal symmetric hearing in the frequencies between $250 \mathrm{~Hz}$ to $8,000 \mathrm{~Hz}$, except one patient with U-shaped symmetric high tone sensory neural hearing loss $(40 \mathrm{~dB}$ at $4,000 \mathrm{~Hz})$. Thirty-two patients were found to have asymmetric sensorineural hearing loss on EHF audiometry. Twenty-eight patients were found to have SSNHL on EHF audiology, while the other 4 did not meet the criteria of SSNHL, as one had a 30-dB difference in only one frequency, another had a $10-\mathrm{dB}$ difference in 2 consecutive frequencies, one had $10-\mathrm{dB}$ difference in 2 nonconsecutive frequencies, and the last had a 10-dB difference in only one frequency. The mean thresholds in the non-affected ear compared with the affected ear are shown on graph 1 . The mean deltas between the sides were between 9.2 and $33 \mathrm{~dB}$ (worse in the affected ear). All patients were treated with an oral steroid (oral prednisone $1 \mathrm{mg} / \mathrm{kg}$ for 7 days).

\section{Discussion}

In the past few decades, the measurement of the EHF audiometry range became possible due to the development of audiometers capable of delivering sounds with sufficient pressure levels, and earphones that can optimally transmit sound through the ear canal. Despite these advances, EHF audiometry is not routinely used in daily clinical practice. Various uses for EHF audiometry have been proposed, including the evaluation of EHF audiometry for early detection of ototoxicity ${ }^{2}$ and as a more sensitive evaluation of noiseinduced hearing loss, ${ }^{3}$ though the latter indication is still controversial. $^{4-6}$ The effect of acoustic trauma on EHF thresholds was investigated in several studies demonstrating great variability of subjects, ${ }^{7,8}$ though Borchgrevnik et al found EHF hearing loss at around $9000 \mathrm{~Hz}$ in these cases. ${ }^{9} \mathrm{~A}$ few studies have also evaluated the auditory status of EHF in serous otitis media and recurrent acute otitis media, demonstrating elevated EHF thresholds as compared with the control group. ${ }^{10-13}$ To the best of our knowledge, this is the largest series evaluating EHF hearing loss in patients presenting with acute tinnitus and intact audiometry results in frequencies of 250 to $8,000 \mathrm{~Hz}$. In chronic tinnitus, no 
pathology will be found in 7.4 to $20 \%$ of the patients using conventional audiometry. ${ }^{14}$ Only a handful of studies have evaluated patients with chronic tinnitus with normal conventional pure tone audiometry utilizing EHF audiometry, with varying results. ${ }^{14-17}$ However, in the present study, we evaluated patients with acute tinnitus. The major pathophysiological difference between acute tinnitus and chronic tinnitus is that chronic tinnitus results from pathological brain activity mainly in the temporal gyrus which presents the humans brain's hearing center. These reported central changes might be the cause of the persistent nature of chronic tinnitus. Changes in brain activity were reported in functional magnetic resonance imaging (MRI) and recently in PET CT demonstrating the central neural changes as a possible cause of chronic tinnitus. However, cochlear or peripheral neural pathology is to be suspected in acute tinnitus as central neural changes require time, and are thereby probably not the pathophysiological etiology of acute tinnitus. Acute tinnitus usually accompanies SSNHL that will be proven by a pathologic conventional hearing loss. Although the pathophysiology of SSNHL is not fully understood, several theories have been proposed including vascular occlusion, membrane breaks, and viral cochleitis or neuritis. The management of acute subjective tinnitus associated with SSNHL includes oral, intravenous, and/or intratympanic administration of corticosteroids as initial therapy. ${ }^{18}$ Therapeutic strategies that improve hearing are reported to have positive effects on tinnitus in most patients with SSNHL together with tinnitus and recently also in patients with acute tinnitus with no pathology on audiometric tests. ${ }^{19}$ The conjugated improvement in both hearing loss and tinnitus in those suffering from both signifies shared etiology; therefore, one should seek for hearing loss in patients presenting with tinnitus and normal conventional audiometry. As the latter encompasses the examination of 250 to $8,000 \mathrm{~Hz}$ only, it seems almost trivial and essential to evaluate hearing in the EHF up to $20000 \mathrm{~Hz}$ to demonstrate the "hidden" SSNHL. This is what was done in our study, proving that tinnitus alone is actually accompanied by EHF SSNHL. The downfalls of our study are its small size and lack of follow-up. However, according to our results, even patients presenting with acute tinnitus and no hearing loss sensation and with normal conventional hearing tests should be regarded as patients with SSNHL, as hearing impairment is observed on EHF audiometry. In view of our results, all patients presenting with acute tinnitus and a normal conventional audiometry should complete EHFaudiometry up to $20,000 \mathrm{~Hz}$ and proceed to the sudden sensory hearing loss management protocol if EHF hearing loss is revealed. Further studies on larger cohorts should be designed to evaluate the effectiveness of SSNHL management protocols on the hereby newly reported EHF hearing loss in patients with acute tinnitus and normal conventional hearing tests.

\section{Conclusion}

Extended high frequency audiometry up to $20,000 \mathrm{~Hz}$ should be completed in all patients presenting with acute tinnitus who have standard audiometry within the normal limits. If hearing impairment is revealed, SSNHL management should be followed.

\section{Conflict of Interests}

The authors have no conflict of interests to declare.

\section{References}

1 Rodríguez Valiente A, Trinidad A, García Berrocal JR, Górriz C, Ramírez Camacho R. Extended high-frequency (9-20. kHz) audiometry reference thresholds in 645 healthy subjects. Int J Audiol 2014;53(08):531-545

2 Fausti SA, Larson VD, Noffsinger D, Wilson RH, Phillips DS, Fowler CG. High-frequency audiometric monitoring strategies for early detection of ototoxicity. Ear Hear 1994;15(03):232-239

3 Somma G, Pietroiusti A, Magrini A, et al. Extended high-frequency audiometry and noise induced hearing loss in cement workers. Am J Ind Med 2008;51(06):452-462

4 Schmuziger N, Brechbuehl M, Probst R. Acoustic measures of lowfrequency noise in extended high-frequency audiometry. J Acoust Soc Am 2007;121(03):EL120-EL124

5 Osterhammel D. High-frequency audiometry and noise-induced hearing loss. Scand Audiol 1979;8(02):85-90

6 Balatsouras DG, Homsioglou E, Danielidis V. Extended highfrequency audiometry in patients with acoustic trauma. Clin Otolaryngol 2005;30(03):249-254

7 Fausti SA, Erickson DA, Frey RH, Rappaport BZ, Schechter MA. The effects of noise upon human hearing sensitivity from 8000 to 20 000. Hz. J Acoust Soc Am 1981;69(05):1343-1347

8 Fausti SA, Erickson DA, Frey RH, Rappaport BZ. The effects of impulsive noise upon human hearing sensitivity (8 to 20 . $\mathrm{kHz}$ ). Scand Audiol 1981;10(01):21-29

9 Borchgrevink HM, Hallmo P, Mair IWS. Extended high-frequency hearing loss from noise exposure. Scientific Basis of Noise-induced Hearing Loss. ThiemeNew York

10 Hassmann-Poznańska E, Goździewski A, Piszcz M, Zajaczkiewicz $\mathrm{H}$, Skotnicka B. [Influence of tympanic membrane changes on immittance and extended frequency audiometric findings]. Otolaryngol Pol 2010;64(05):307-312

11 Ahonen JE, McDermott JC. Extended high-frequency hearing loss in children with cleft palate. Audiology 1984;23(05):467-476

12 Sharma D, Munjal SK, Panda NK. Extended high frequency audiometry in secretory otitis media. Indian J Otolaryngol Head Neck Surg 2012;64(02):145-149

13 Laitila P, Karma P, Sipilä M, Manninen M, Rakho T. Extended high frequency hearing and history of acute otitis media in 14-year-old children in Finland. Acta Otolaryngol Suppl 1997;529:27-29

14 Shim HJ, Kim SK, Park CH, et al. Hearing abilities at ultra-high frequency in patients with tinnitus. Clin Exp Otorhinolaryngol 2009;2(04):169-174

15 Barnea G, Attias J, Gold S, Shahar A. Tinnitus with normal hearing sensitivity: extended high-frequency audiometry and auditorynerve brain-stem-evoked responses. Audiology 1990;29(01):36-45

16 Kim DK, Park SN, Kim HM, et al. Prevalence and significance of highfrequency hearing loss in subjectively normal-hearing patients with tinnitus. Ann Otol Rhinol Laryngol 2011;120(08):523-528

17 Fabijańska A, Smurzyński J, Hatzopoulos S, et al. The relationship between distortion product otoacoustic emissions and extended high-frequency audiometry in tinnitus patients. Part 1 : normally hearing patients with unilateral tinnitus. Med Sci Monit 2012;18 (12):CR765-CR770

18 Hoare DJ, Kowalkowski VL, Kang S, Hall DA. Systematic review and meta-analyses of randomized controlled trials examining tinnitus management. Laryngoscope 2011;121(07):1555-1564

19 An YH, Yu KK, Kwak MY, Yoon SW, Shim HJ. Prognostic factors for the outcomes of intratympanic dexamethasone in the treatment of acute subjective tinnitus. Otol Neurotol 2014;35(08):1330-1337 